\title{
Retinal thickness among normal myopic Filipinos using spectral domain optical coherence tomography
}

\author{
Camille Elaine Zabala, Jubaida Mangondato-Aquino, \\ Jose Ma. Martinez, John Mark de Leon \\ Department of Health Eye Center, East Avenue Medical Center, Quezon City, \\ Philippines
}

\begin{abstract}
Purpose: To determine mean macular and retinal nerve fiber layer (RNFL) thickness of myopic Filipinos using spectral domain optical coherence tomography (SD-OCT) and to evaluate influence of age, gender, and degree of myopia.

Design: Observational clinic-based cohort

Methods: Participants were divided into two groups: low-moderate myopia [spherical equivalent (SE) - $0.50 \mathrm{D}$ to $-6.00 \mathrm{D}$ ] and high-pathologic myopia (SE $<-6.00 \mathrm{D}$ and $\mathrm{AL}>$ $26.5 \mathrm{~mm}$ ). Subgroup analyses between low myopia (refraction $<-3.00 \mathrm{D}$ or less) and moderate myopia (> $-3.00 \mathrm{D}$ to $-6.00 \mathrm{D})$, and high myopia (> $-6.00 \mathrm{D}$ to $-8.00 \mathrm{D})$ and pathologic myopia (more than $-8.00 \mathrm{D}$ ) were done. Macular and RNFL thickness were measured by a SD-OCT and axial length (AL) with non-contact biometry.

Results: Of 156 eyes, 88/156 (56\%) had low-moderate myopia, 68/156 (44\%) had high-pathologic myopia. There were 67/156 (43\%) male and 89/156 (57\%) female subjects. Mean central foveal subfield thickness measurements were $264 \pm 24 \mu \mathrm{m}$ for low myopia, $258 \pm 17 \mu \mathrm{m}$ for moderate myopia, $253 \pm 25 \mu \mathrm{m}$ for high myopia, and $218 \pm 48$ $\mu \mathrm{m}$ for pathologic myopia. Mean RNFL thickness measurements were $105.62 \pm 3.89 \mu \mathrm{m}$ for low myopia, $97.6 \pm 2.45 \mu \mathrm{m}$ for moderate myopia, $85.9 \pm 3.87 \mu \mathrm{m}$ for high myopia, and $75.14 \pm 3.89 \mu \mathrm{m}$ for pathologic myopia. Average SE $(p<0.0001)$ decreased while AL $(p<0.0001)$ increased with more myopia. Myopia and age significantly affected macular and RNFL thickness parameters except for the following where only the degree of myopia was a significant factor: central foveal, temporal parafoveal, nasal perifoveal, inferior and nasal RNFL thicknesses.

Conclusion: Retinal SD-OCT thickness measurements decreased with increasing level of myopia and age. Central foveal, temporal parafoveal, nasal perifoveal, inferior and nasal RNFL thicknesses may be more appropriate SD-OCT parameters among myopic Filipino patients to monitor for glaucoma since they may be less influenced by age.
\end{abstract}

Correspondence: John Mark de Leon, Department of Health Eye Center, East Avenue Medical Center, East Avenue, Diliman, Quezon City, Philippines.

E-mail:jmarkmd1@yahoo.com 
Keywords: Filipino, macular thickness, myopia, retinal nerve fiber layer thickness, spectral-domain OCT

Myopia is identified by the World Health Organization as one of the leading causes of visual disability and the most common ocular disorder among children and adults worldwide. In the Beaver Dam and Baltimore Eye Studies, the prevalence rates of myopia among adults were $22.7 \%$ and $26.2 \%$, respectively. ${ }^{1}$ These prevalence rates were seen to be higher for Southeast Asian countries at $31 \%$ to $40 \%$, with an exponential growth observed during recent years. ${ }^{2-4} \mathrm{~A}$ clinic-based study in the Philippines revealed a myopia prevalence rate of 59\%; however, this was derived from a patient population at a refractive/cataract screening centre. ${ }^{5}$ With the current statistics and the projected increase in prevalence rates, myopia poses a large public health impact due to the visual difficulties and other potentially blinding ocular conditions associated with it. By 2030, the projected rate is estimated to be more than $50 \%$. This translates to a significant number of people with potential visual impairment inherent with the condition. ${ }^{6-8}$

Myopic individuals may have structural changes in the eye brought about by the increased axial elongation inherent in the disease process. Moreover, myopia has been established to have associations with other ocular diseases such as glaucoma, optic nerve abnormalities and a myriad of chorioretinal abnormalities. ${ }^{9}$ Diagnosis of these conditions becomes a challenge to the examiner because of structural factors. For glaucoma, the tilting of the optic nerve, large ovalness index, deformation of the disc, shallow and large cup, large peripapillary crescent and occasional optic disc hypoplasia of highly myopic subjects impede accurate diagnosis. ${ }^{10}$ The recent use of retinal nerve fibre layer (RNFL) thickness analysis using spectral-domain optical coherence tomography (SD-OCT) had aided clinicians in distinguishing glaucomatous damage. However, SD-OCT's may falsely diagnose normal myopic patients with glaucoma since their normative data were collected from normal eyes with low to no myopia. ${ }^{11}$ Similarly, the use of SD-OCT macular examination for the diagnosis and monitoring of diseases of the macula may give erroneous results for myopic individuals when compared with age-matched individuals with normal axial length ( $A L)$.

The establishment of mean local data for macula and RNFL thickness parameters among myopic patients can be used for comparison in the clinical setting in order to diagnose glaucoma and other macular diseases more accurately and to monitor its progression.

In a meta-analysis done, myopia was defined in most studies as having a spherical equivalent (SE) of $-0.50 \mathrm{D}$ or less $(58.7 \%$ of all included studies). 
$\mathrm{AL}>26.5 \mathrm{~mm}$. Pathologic myopia was defined as an SE of less than $-8.00 \mathrm{D}$ or an $A L \geq 32.5 \mathrm{~mm} .^{8,12,13}$ Due to the increased $A L$ in myopia, there are several ocular structures that may be altered. Studies have demonstrated the direct relationship between retinal thickness and increased AL due to thinning of the choroid and sclera and elongation of photoreceptor outer segments. ${ }^{14}$ Previous investigations have also revealed that the optic disc and the peripapillary atrophy start to enlarge at about an $\mathrm{AL}$ of $\sim 26.5 \mathrm{~mm}$ and that the prevalence of myopic retinopathy and glaucomatous optic neuropathy steeply increased beyond this value. ${ }^{13}$ There are several ocular diseases associated with myopia. These diseases may develop in part due to the increased $A L$ and the concurrent structural changes induced within the eye. Previous population-based and hospital-based studies have revealed that axial myopia, in particular high axial myopia, is a risk factor for the development of several vitreoretinal pathologies. Myopic patients are also at risk to develop rhegmatogenous retinal detachment and subretinal neovascularization. In addition, they were also seen to have optic nerve changes such as the presence of optic nerve crescents and peripapillary atrophy. ${ }^{7,13}$ Because of the structural changes in the eye that occur concomitantly with myopia, it is a challenge for clinicians to diagnose some clinical entities such as glaucoma or macular thinning. For instance, the advent of SD-OCT has been useful in identifying damage. However, a significant number of normal myopic eyes might have been flagged as abnormal primarily because the normative data were derived from eyes with normal to no myopia. Furthermore, the normative data of the OCT machines derive their values mostly from other races, and interracial differences in measurements are not taken into consideration. As a result, it is sometimes difficult for Filipino ophthalmologists to decide whether abnormal values on macular and RNFL thickness are reliable.

The main objective of our study is to determine the mean values for macular and RNFL thickness measures among adult myopic Filipinos. Specific objectives are as follows: (1) to describe the demographic characteristics of patients with myopia, (2) to compare average RNFL and macular thickness measurements across the different degrees of myopia, (3) to evaluate the effects of age and gender on RNFL and macular thickness and (4) to provide mean values for macular and RNFL thickness measurements among the different degrees of myopia.

\section{Materials and methods}

\section{Study population}

This cross-sectional, observational study was conducted at an eye centre of a tertiary government hospital in Quezon City, Metro Manila, Philippines. All the patients seen consecutively at the outpatient department from August 22 to 
September 2, 2016, were screened. Patients were included in the study if they satisfy the following inclusion criteria: age $>18$ years, best-corrected visual acuity (BCVA) better than or equal to $20 / 40$, no history of previous intraocular and refractive surgery and a refraction of $-0.50 \mathrm{D}$ or less by automated kerato-refractometer (GRK1 Auto Ref/Keratometer, Ryusyo Industrial Co., Ltd, Osaka, Japan). Patients with other ocular diseases such as glaucoma, chorioretinitis, retinopathies and other neurologic diseases that may alter the optic nerve head, RNFL and the macula were also excluded from this study.

All participants had provided full and informed consent prior to participation in this study. This study was approved by the Institutional Ethics Review Board of the East Avenue Medical Center. All aspects of this study adhered to the tenets of the Declaration of Helsinki.

\section{Clinical examination and myopia classification}

All patients underwent complete history-taking, visual acuity testing, colour testing using Ishihara plates, slit-lamp biomicroscopy (Haag-Streit ${ }^{\oplus}$, Koenz, Switzerland), intraocular pressure measurement using Goldmann applanation tonometry (Haag-Streit ${ }^{\circledR}$, Koenz, Switzerland), indirect ophthalmoscopy (Keeler ${ }^{\circledast}$ Ophthalmic Instruments, Windsor, United Kingdom) using $20 \mathrm{D}$ and $90 \mathrm{D}$ lenses (Volk ${ }^{\circledR}$ Optical, Inc., Mentor, OH, USA) and objective refraction. All examinations were performed by an ophthalmology resident who was not part of this study.

All recruited patients underwent macular and RNFL thickness measurement using an SD-OCT (Spectralis', Heidelberg Engineering, Heidelberg, Germany) on the same day. Only those patients whose scans had good centration and quality scores $\geq 20$ were used for analysis. The thickness of the RNFL, defined as the area between the inner margins of the internal limiting membrane and the outer segment of the RNFL was measured around the optic nerve. The measurement was derived from averaging 16 consecutive circular B-scans, each with a diameter of $3.5 \mathrm{~mm}$. The built-in tracking system (TruTrack image alignment software) was used to maintain fixation on target areas despite eye movement. The Spectralis software, version 5.3.3.0, was used. Following this, the OCT volume scan was done on a $20 \times 20$ degree cube with 49 raster lines, each containing 1,064 pixels, separated by 120 microns. All scans were performed by one technician who is not aware of the patient's screening results, who reviewed each of the scans. Macular thickness measurements were reported using a modified Early Treatment of Diabetic Retinopathy Study (ETDRS) macular map. The ETDRS map has central 1-mm foveal field, surrounded an inner (parafoveal) and outer (perifoveal) subfields having diameters of $3 \mathrm{~mm}$ and $6 \mathrm{~mm}$, respectively. AL measurements were done using an optical biometry (IOL Master ${ }^{\circledR} 500$, Carl Zeiss Meditec, Oberkochen, Germany) by a single technician who was not aware of the screening results. 
Retinal thickness among normal myopic Filipinos

Table 1. Patient demographics

\begin{tabular}{|c|c|c|c|c|c|}
\hline & \multicolumn{4}{|c|}{ Myopia degree } & \multirow{3}{*}{$p$} \\
\hline & Low & Moderate & High & Pathologic & \\
\hline & $(n=55)$ & $(n=33)$ & $(n=27)$ & $(n=41)$ & \\
\hline \multicolumn{6}{|l|}{ Age (years) } \\
\hline Mean \pm SD & $36 \pm 13$ & $31 \pm 10$ & $41 \pm 13$ & $43 \pm 14$ & $0.001^{\mathrm{a}}$ \\
\hline Median (IQR) & $31(14)$ & $30(8)$ & $37(23)$ & $42(28)$ & \\
\hline \multicolumn{6}{|c|}{ Age (years), $n(\%)$} \\
\hline $19-40$ & $40(73 \%)$ & $31(29 \%)$ & $17(63 \%)$ & $18(44 \%)$ & $0.001^{a}$ \\
\hline $41-60$ & $10(18 \%)$ & $1(3 \%)$ & $8(30 \%)$ & $19(46 \%)$ & \\
\hline$>60$ & $5(42 \%)$ & $1(8 \%)$ & $2(17 \%)$ & $4(33 \%)$ & \\
\hline \multicolumn{6}{|l|}{ Gender, $n(\%)$} \\
\hline Male & $30(54 \%)$ & $10(30 \%)$ & $11(41 \%)$ & $16(39 \%)$ & 0.140 \\
\hline Female & $25(46 \%)$ & $23(70 \%)$ & $16(59 \%)$ & $25(61 \%)$ & \\
\hline \multicolumn{6}{|c|}{$\begin{array}{l}\text { Spherical } \\
\text { equivalent (D) }\end{array}$} \\
\hline Mean \pm SD & $-1.7 \pm 0.8$ & $-4.1 \pm 0.5$ & $-6.8 \pm 0.6$ & $-13.7 \pm 4.2$ & $0.000^{\mathrm{a}}$ \\
\hline Median (IQR) & $-2.0(1.0)$ & $-4.0(0.75)$ & $-6.8(0.8)$ & $-12.8(5.9)$ & \\
\hline \multicolumn{6}{|c|}{ Axial length (mm) } \\
\hline Mean \pm SD & $24.3 \pm 0.7$ & $25.7 \pm 0.6$ & $26.9 \pm 0.9$ & $29.4 \pm 2.3$ & $0.000^{\mathrm{a}}$ \\
\hline Median (IQR) & $24.4(0.9)$ & $25.8(0.9)$ & $26.7(0.4)$ & $28.4(3.0)$ & \\
\hline
\end{tabular}

aSignificant at $5 \%$ level

IQR: interquartile range; SD: standard deviation

Participants were divided into two groups for analysis: low-to-moderate myopia ( $\mathrm{SE}-0.50 \mathrm{D}$ to $-6.00 \mathrm{D}$ ) and high-pathologic myopia (SE less than $-6.00 \mathrm{D}$ and $A L>26.5 \mathrm{~mm}$ ). Furthermore, subgroup analyses between low myopia (refraction less than $-3.00 \mathrm{D}$ ) and moderate myopia (more than $-3.00 \mathrm{D}$ to $-6.00 \mathrm{D}$ ) and high myopia (more than $-6.00 \mathrm{D}$ to $-8.00 \mathrm{D}$ ) and pathologic myopia (more than $-8.00 \mathrm{D}$ ) were done.

\section{Statistical analysis}

Data of evaluable patients were encoded in MS Excel spreadsheets. Data processing and statistical analyses were done using IBM SPSS v. 20.0 statistical software (IBM Corporation, New York, USA). Descriptive statistics were presented 
Table 2. Visual acuity

\begin{tabular}{|l|l|l|l|l|l|}
\hline & \multicolumn{3}{|l|}{ Myopia degree } & \\
\hline & Low & Moderate & High & Pathologic & \multirow{2}{*}{$p$} \\
\cline { 2 - 5 } & $(\boldsymbol{n}=\mathbf{5 5})$ & $(\boldsymbol{n}=\mathbf{3 3})$ & $(\boldsymbol{n}=\mathbf{2 7})$ & $(\boldsymbol{n}=\mathbf{4 1})$ & \\
\hline $\begin{array}{l}\text { Visual } \\
\begin{array}{l}\text { acuity(LogMar) } \\
\text { Mean } \pm \text { SD }\end{array}\end{array}$ & $0.04 \pm 0.01$ & $0.07 \pm 0.13$ & $0.11 \pm 0.13$ & $0.45 \pm 0.22$ & $0.000^{\mathrm{a}}$ \\
\hline Median (IQR) & $0.00(0.00)$ & $0.00(0.10)$ & $0.10(0.20)$ & $0.40(0.40)$ & \\
\hline
\end{tabular}

aSignificant at $5 \%$ level

IQR: interquartile range; SD: standard deviation

as mean \pm SD and median (IQR) and as $n(\%)$ for categorical data. Checks for homogeneity of sample population and normality assumption were performed. Kruskal-Wallis test was performed to determine whether the thickness measurements differed across levels of myopia. Stepwise multiple regression analysis was used to provide prediction equations of each macular and RNFL thickness measures considering effects of levels of myopia, age and gender. Regression models based on four levels of myopia (low, moderate, high, and pathologic) and two levels (low-to-moderate and high-to-pathologic) were generated and compared. Diagnostic checks were performed in selecting the best-fit regression models. Normative values of macular and RNFL thickness measures by level of myopia were generated based on the final regression equations generated using four levels of myopia. Statistical significance was based on $p$-value $\leq 0.05$.

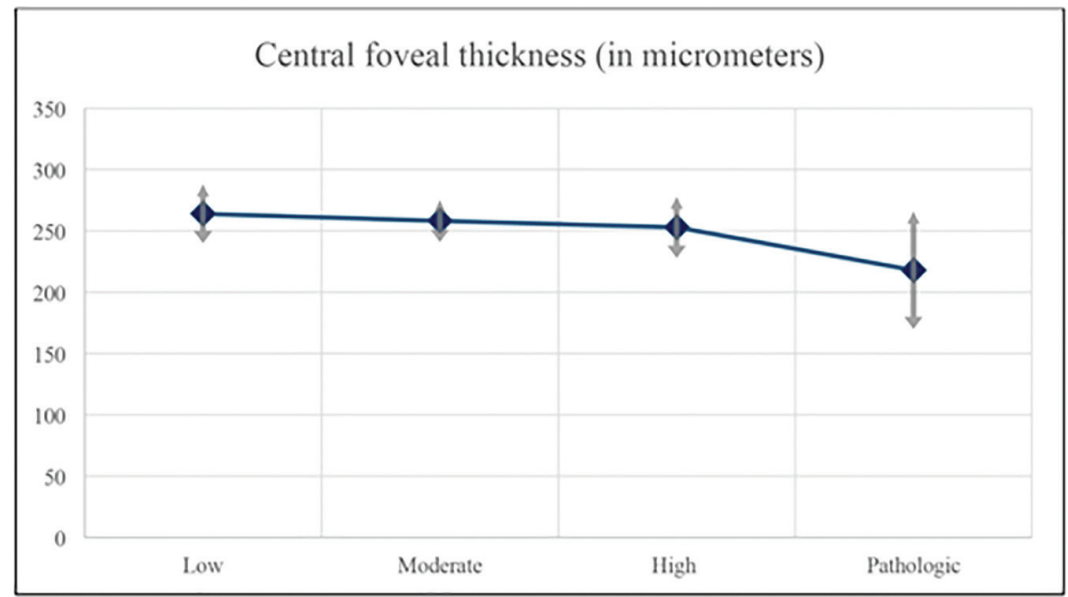

Fig. 1. Mean central foveal thickness across different degrees of myopia 
RNFL thickness measurements (in millimeters)

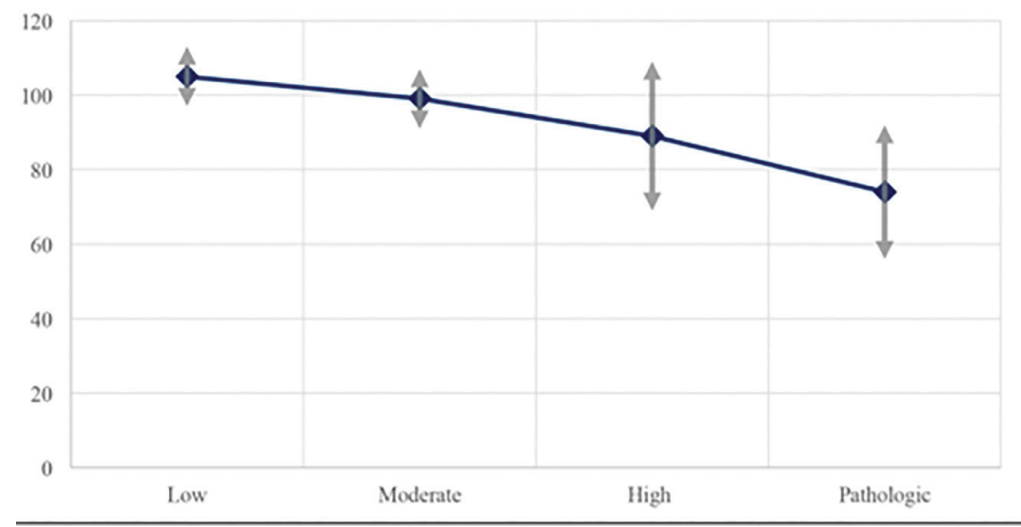

Fig. 2. Mean RNFL thickness across different degrees of myopia

\section{Results}

A total of 175 eyes were included in our study. However, some participants were excluded in the analysis due to the presence of concurrent ocular conditions such as glaucoma (four eyes), previous history of intraocular surgery (eight eyes) and refractive surgery (two eyes). Five scans were also excluded due to poor signal strength.

Table 1 shows the number and percentage of eyes for each of the degrees of myopia. The average age of patients was 32 years (IQR $=23$; range from 19 to 67 years old). There were $67 / 156$ (43\%) male and 89/156 (57\%) female subjects. Majority were unilateral cases $(52 \% ; p=0.497)$. Patients with pathologic myopia were oldest (42 years; $p=0.001$ ). Majority of patients with low $(73 \%)$, moderate (29\%) or high myopia (63\%) belonged to the 19 to 40 years age group $(p=0.001)$. Most patients with low (54\%) myopia were male, while those with moderate, high or pathologic myopia were female $(p=0.140)$. Average SE $(p=0.000)$ and AL $(p=0.000)$ decreased with level of myopia.

It was observed that average BCVA decreased with the level of myopia $(p=0.000$; Table 2). All patients had normal colour vision by Ishihara testing. For the macula, the mean central foveal subfield thickness was the thinnest among all the parameters at $249 \pm 33 \mu \mathrm{m}$. Figure 1 summarizes the mean central foveal thickness measurements and reflects the decrease in the means as the degree of myopia progresses ( $p<0.0001$; Table 3 ). There were generally thicker measurements for the parafoveal and perifoveal regions. The mean circumpapillary RNFL thickness global average was $92.51 \pm 18.60 \mu \mathrm{m}$. Figure 2 summarizes the circumpapillary RNFL thickness and shows that RNFL measurements decreases with level of myopia ( $p<0.0001$; Table 3 ). Regression equations were generated 
Zabala, Mangondato-Aquino, Martinez, de Leon

Table 3. Comparison between different OCT parameters

\begin{tabular}{|c|c|c|c|c|c|}
\hline \multirow{3}{*}{ Thickness ( $\mu \mathrm{m})$} & \multicolumn{4}{|c|}{ Myopia degree } & \multirow{3}{*}{$p$} \\
\hline & Low & Moderate & High & Pathologic & \\
\hline & $(n=55)$ & $(n=33)$ & $(n=27)$ & $(n=41)$ & \\
\hline $\begin{array}{l}\text { Central foveal } \\
\text { Mean } \pm \text { SD } \\
\text { Median (IQR) }\end{array}$ & $\begin{array}{l}264 \pm 24 \\
263(33)\end{array}$ & $\begin{array}{l}258 \pm 17 \\
261(19)\end{array}$ & $\begin{array}{l}253 \pm 25 \\
261(42)\end{array}$ & $\begin{array}{l}218 \pm 48 \\
232(88)\end{array}$ & $0.000^{\mathrm{a}}$ \\
\hline $\begin{array}{l}\text { Parafoveal superior } \\
\text { Mean } \pm \text { SD } \\
\text { Median (IQR) }\end{array}$ & $\begin{array}{l}343 \pm 14 \\
343(23)\end{array}$ & $\begin{array}{l}337 \pm 15 \\
336(17)\end{array}$ & $\begin{array}{l}320 \pm 19 \\
318(35)\end{array}$ & $\begin{array}{l}281 \pm 40 \\
288(80)\end{array}$ & $0.000^{\mathrm{a}}$ \\
\hline $\begin{array}{l}\text { Parafoveal inferior } \\
\text { Mean } \pm \text { SD } \\
\text { Median (IQR) }\end{array}$ & $\begin{array}{l}337 \pm 12 \\
336(18)\end{array}$ & $\begin{array}{l}335 \pm 13 \\
333(17)\end{array}$ & $\begin{array}{l}316 \pm 21 \\
316(43)\end{array}$ & $\begin{array}{l}277 \pm 38 \\
274(70)\end{array}$ & $0.000^{\mathrm{a}}$ \\
\hline $\begin{array}{l}\text { Parafoveal nasal } \\
\text { Mean } \pm \text { SD } \\
\text { Median (IQR) } \\
\end{array}$ & $\begin{array}{l}344 \pm 15 \\
341(21) \\
\end{array}$ & $\begin{array}{l}339 \pm 16 \\
338(18)\end{array}$ & $\begin{array}{l}322 \pm 20 \\
322(37)\end{array}$ & $\begin{array}{l}282 \pm 43 \\
300(88)\end{array}$ & $0.000^{\mathrm{a}}$ \\
\hline $\begin{array}{l}\text { Parafoveal temporal } \\
\text { Mean } \pm \text { SD } \\
\text { Median (IQR) }\end{array}$ & $\begin{array}{l}328 \pm 13 \\
326(18)\end{array}$ & $\begin{array}{l}322 \pm 16 \\
321(21)\end{array}$ & $\begin{array}{l}311 \pm 18 \\
313(37)\end{array}$ & $\begin{array}{l}279 \pm 32 \\
280(54)\end{array}$ & $0.000^{\mathrm{a}}$ \\
\hline $\begin{array}{l}\text { Perifoveal superior } \\
\text { Mean } \pm \text { SD } \\
\text { Median (IQR) }\end{array}$ & $\begin{array}{l}304 \pm 15 \\
304(19)\end{array}$ & $\begin{array}{l}303 \pm 10 \\
304(15)\end{array}$ & $\begin{array}{l}290 \pm 15 \\
287(15)\end{array}$ & $\begin{array}{l}273 \pm 24 \\
277(29)\end{array}$ & $0.000^{\mathrm{a}}$ \\
\hline $\begin{array}{l}\text { Perifoveal inferior } \\
\text { Mean } \pm \text { SD } \\
\text { Median (IQR) }\end{array}$ & $\begin{array}{l}291 \pm 14 \\
292(20) \\
\end{array}$ & $\begin{array}{l}288 \pm 9 \\
289(9) \\
\end{array}$ & $\begin{array}{l}274 \pm 17 \\
277(30) \\
\end{array}$ & $\begin{array}{l}260 \pm 25 \\
263(31) \\
\end{array}$ & $0.000^{\mathrm{a}}$ \\
\hline $\begin{array}{l}\text { Perifoveal nasal } \\
\text { Mean } \pm \text { SD } \\
\text { Median (IQR) }\end{array}$ & $\begin{array}{l}320 \pm 15 \\
319(25)\end{array}$ & $\begin{array}{l}316 \pm 13 \\
315(20)\end{array}$ & $\begin{array}{l}303(16) \\
301(29)\end{array}$ & $\begin{array}{l}272 \pm 48 \\
273(44)\end{array}$ & $0.000^{\mathrm{a}}$ \\
\hline $\begin{array}{l}\text { Perifoveal temporal } \\
\text { Mean } \pm \text { SD } \\
\text { Median (IQR) }\end{array}$ & $\begin{array}{l}289 \pm 18 \\
288(16)\end{array}$ & $\begin{array}{l}290 \pm 16 \\
287(17) \\
\end{array}$ & $\begin{array}{l}275 \pm 18 \\
275(15) \\
\end{array}$ & $\begin{array}{l}264 \pm 26 \\
269(34)\end{array}$ & $0.000^{\mathrm{a}}$ \\
\hline $\begin{array}{l}\text { RNFL global average } \\
\text { Mean } \pm \text { SD } \\
\text { Median (IQR) }\end{array}$ & $\begin{array}{l}105 \pm 8 \\
103(11)\end{array}$ & $\begin{array}{l}99 \pm 8 \\
99(12)\end{array}$ & $\begin{array}{l}89 \pm 20 \\
92(17)\end{array}$ & $\begin{array}{l}74 \pm 18 \\
72(25)\end{array}$ & $0.000^{\mathrm{a}}$ \\
\hline $\begin{array}{l}\text { RNFL superior } \\
\text { Mean } \pm \text { SD } \\
\text { Median (IQR) }\end{array}$ & $\begin{array}{l}131 \pm 16 \\
130(22)\end{array}$ & $\begin{array}{l}128 \pm 14 \\
129(14)\end{array}$ & $\begin{array}{l}111 \pm 17 \\
111(21)\end{array}$ & $\begin{array}{l}78 \pm 28 \\
79(47)\end{array}$ & $0.000^{\mathrm{a}}$ \\
\hline
\end{tabular}


Retinal thickness among normal myopic Filipinos

\begin{tabular}{|c|c|c|c|c|c|}
\hline \multirow{3}{*}{ Thickness $(\mu \mathrm{m})$} & \multicolumn{4}{|c|}{ Myopia degree } & \multirow{3}{*}{$p$} \\
\hline & Low & Moderate & High & Pathologic & \\
\hline & $(n=55)$ & $(n=33)$ & $(n=27)$ & $(n=41)$ & \\
\hline $\begin{array}{l}\text { RNFL inferior } \\
\text { Mean } \pm \text { SD } \\
\text { Median (IQR) }\end{array}$ & $\begin{array}{l}140 \pm 24 \\
138(23)\end{array}$ & $\begin{array}{l}125 \pm 18 \\
128(19)\end{array}$ & $\begin{array}{l}121 \pm 16 \\
121(16)\end{array}$ & $\begin{array}{l}82 \pm 31 \\
85(44)\end{array}$ & $0.000^{\mathrm{a}}$ \\
\hline $\begin{array}{l}\text { RNFL nasal } \\
\text { Mean } \pm \text { SD } \\
\text { Median (IQR) }\end{array}$ & $\begin{array}{l}78 \pm 15 \\
76(12)\end{array}$ & $\begin{array}{l}71 \pm 19 \\
71(24)\end{array}$ & $\begin{array}{l}67 \pm 17 \\
70(21)\end{array}$ & $\begin{array}{l}51 \pm 20 \\
45(29)\end{array}$ & $0.000^{\mathrm{a}}$ \\
\hline $\begin{array}{l}\text { RNFL temporal } \\
\text { Mean } \pm \text { SD } \\
\text { Median (IQR) }\end{array}$ & $\begin{array}{l}75 \pm 8 \\
75(10)\end{array}$ & $\begin{array}{l}75 \pm 11 \\
77(13)\end{array}$ & $\begin{array}{l}77 \pm 16 \\
76(21)\end{array}$ & $\begin{array}{l}68 \pm 27 \\
69(37)\end{array}$ & 0.137 \\
\hline
\end{tabular}

aSignificant at $5 \%$ level

IQR: interquartile range; SD: standard deviation

and compared based on four levels of myopia (low, moderate, high and pathologic and two levels); however, based on the results of diagnostic checks, the final regression models were those generated using four levels of myopia. Multiple regression analysis showed that level of myopia and age influence macular and RNFL thickness parameters except for central foveal subfield thickness, temporal parafoveal thickness, nasal perifoveal thickness, inferior RNFL thickness and nasal RNFL thickness where only the level of myopia was a significant factor (Fig. 3). No regression model could be generated for temporal RNFL thickness since the level of myopia, age and gender were not significant factors. Table 4 shows the reference range of macular and RNFL thickness measurements per level of myopia with correction for age.

\section{Discussion}

In our study among normal myopic Filipinos, the mean central foveal subfield thickness measurement was $249 \pm 33 \mu \mathrm{m}$. This was lower than the mean of $270 \pm 22.5 \mu \mathrm{m}$ derived using the Spectralis ${ }^{\circ}$ SD-OCT from a previous study, whose subjects comprised mostly of a Caucasian population with no-to-low myopia. ${ }^{15}$ It was closely comparable to the mean value of $250.83 \pm 17.04 \mu \mathrm{m}$ obtained also using the Spectralis ${ }^{\circ}$ SD-OCT in a previous study from the same institution; however, the subjects were normal Filipinos with no-to-low myopia (unpublished, Dans et al., oral presentation, Philippine Academy of Ophthalmology 2015). These findings may suggest differences between the retinal thickness measurements across different races.

We observed that macular thickness parameters were thinnest at the central foveal subfield, with increasing measurements for the parafoveal and perifoveal 
Table 4. Macular and RNFL thickness parameters by degrees of myopia

\begin{tabular}{|c|c|c|}
\hline Thickness parameter & Degree of myopia & Range ( $\mu \mathrm{m})$ \\
\hline \multirow{4}{*}{ Central foveal } & Low & 267.91-268.61 \\
\hline & Moderate & $253.84-254.08$ \\
\hline & High & $239.45-239.87$ \\
\hline & Pathologic & $224.79-226.61$ \\
\hline \multirow{4}{*}{ Parafoveal superior } & Low & $341.85-352.75$ \\
\hline & Moderate & $327.14-333.74$ \\
\hline & High & $302.87-313.81$ \\
\hline & Pathologic & $281.87-293.63$ \\
\hline \multirow[t]{4}{*}{ Parafoveal inferior } & Low & $336.89-348.21$ \\
\hline & Moderate & $322.72-329.58$ \\
\hline & High & $298.83-310.05$ \\
\hline & Pathologic & $278.43-290.17$ \\
\hline \multirow[t]{4}{*}{ Parafoveal nasal } & Low & $342.65-354.09$ \\
\hline & Moderate & $328.24-335.12$ \\
\hline & High & $304-315.22$ \\
\hline & Pathologic & $283.05-295.03$ \\
\hline \multirow[t]{4}{*}{ Parafoveal temporal } & Low & 331.74-332.14 \\
\hline & Moderate & $316.18-317.40$ \\
\hline & High & $300.55-300.85$ \\
\hline & Pathologic & 284.75-285.95 \\
\hline \multirow[t]{4}{*}{ Perifoveal superior } & Low & $299.85-312.61$ \\
\hline & Moderate & 295.74-303.48 \\
\hline & High & 280.25-293.33 \\
\hline & Pathologic & $268.96-282.14$ \\
\hline \multirow[t]{4}{*}{ Perifoveal inferior } & Low & $288.57-296.49$ \\
\hline & Moderate & $282.07-286.87$ \\
\hline & High & $268.46-276.58$ \\
\hline & Pathologic & $257.22-265.90$ \\
\hline
\end{tabular}


Retinal thickness among normal myopic Filipinos

\begin{tabular}{|c|c|c|}
\hline Thickness parameter & Degree of myopia & Range $(\mu \mathrm{m})$ \\
\hline \multirow{4}{*}{ Perifoveal nasal } & Low & $324.04-324.46$ \\
\hline & Moderate & $308.64-308.82$ \\
\hline & High & 293.15-293.45 \\
\hline & Pathologic & 277.29-278.99 \\
\hline \multirow[t]{4}{*}{ Perifoveal temporal } & Low & 290.62-291.74 \\
\hline & Moderate & $282.45-287.55$ \\
\hline & High & $270.69-279.01$ \\
\hline & Pathologic & $261.05-270.15$ \\
\hline \multirow[t]{4}{*}{ RNFL global average } & Low & $101.73-109.51$ \\
\hline & Moderate & $95.15-100.05$ \\
\hline & High & $82.03-89.77$ \\
\hline & Pathologic & $71.25-79.03$ \\
\hline \multirow[t]{4}{*}{ RNFL superior } & Low & $127.65-142.03$ \\
\hline & Moderate & 117.21-126.13 \\
\hline & High & $94.46-108.98$ \\
\hline & Pathologic & $76.8-90.7$ \\
\hline \multirow[t]{4}{*}{ RNFL inferior } & Low & 142.54-143.24 \\
\hline & Moderate & $124.64-124.90$ \\
\hline & High & 106.42-106.72 \\
\hline & Pathologic & $88.15-89.31$ \\
\hline \multirow[t]{4}{*}{ RNFL nasal } & Low & 78.62-79.06 \\
\hline & Moderate & $70.34-70.60$ \\
\hline & High & $61.94-62.22$ \\
\hline & Pathologic & $53.43-54.19$ \\
\hline
\end{tabular}

areas. This is consistent with the normal anatomic contour as previously reported in OCT studies of the normal macula among Caucasians. ${ }^{16,17}$

In terms of the circumpapillary RNFL thickness measurements, the mean global average derived in our study was $92.51 \pm 18.60 \mu \mathrm{m}$. This was lower than the mean measurement of $100.05 \pm 6.84 \mu \mathrm{m}$ using the same OCT device from a previous study done among normal healthy Koreans and comparable to the mean of $92.7 \pm 7.20 \mu \mathrm{m}$ observed among normal healthy Caucasians. Alasil et al. observed that Asians had thicker RNFL measurements than their Caucasian and 


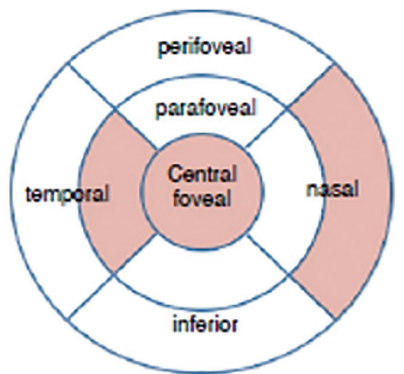

Macular measurements

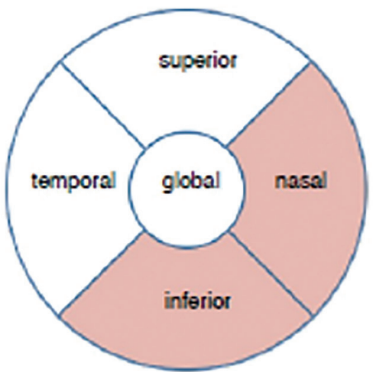

RNFL measurements

Fig. 3. Degree of myopia and age significantly affected thickness parameters, except for those areas highlighted in red (areas only affected by the degree of myopia).

Hispanic counterparts. ${ }^{21}$ The individual RNFL quadrant measurements, however, were all thinner than normal Koreans and Caucasians. ${ }^{18-21}$ The inferior circumpapillary RNFL quadrants had the thickest measurements, followed by the superior, temporal and then nasal quadrants. This slightly deviates from the established ISNT rule, where the inferior was thickest, followed by the superior, nasal and then temporal quadrants. An explanation might be the presence of technical difficulties in measuring the peripapillary area due to structural changes brought about by axial myopia. These may include the tilting of the optic nerve, the presence of peripapillary atrophy and a marked elongation and corresponding thinning of the peripapillary scleral flange. ${ }^{13,18,19}$

In general, there was a negative correlation between all macular and RNFL thickness measurements and degree of myopia based on AL. This could be explained by the mechanical stretching of the eyeball that occurs with myopia, which consequently leads to thinning of ocular structures including the retina. Histologically, thinning of the macula in axial myopia is believed to be caused by the secondary defects in the Bruch's membrane that occur with the condition. This was also supported by histologic evidence of the attenuation or lack of the retinal pigment epithelium cells, choriocapillaries, the large choroidal vessel layer and photoreceptors among pathologic axial myopic patients. ${ }^{13}$

Gender was not a contributing factor in terms of macular and RNFL thickness. This was also demonstrated by previous studies done by Tewari et al. and Grover et al. ${ }^{15}$ In contradistinction, some studies done by Song et al., Wong et al. and Appatukkan et al. revealed that men had thicker macular measurements. ${ }^{20}$ They even attributed the higher incidence of macular holes among women to this particular finding, but this was not evident in this study. This was also evident in a previous study among Filipinos, where male subjects had thicker central macular thickness compared with females (unpublished, Dans et al., oral presentation, Philippine Academy of Ophthalmology 2015). 
There was also a negative correlation between retinal thickness measurements and age, except for central foveal subfield thickness, temporal parafoveal thickness, nasal perifoveal thickness and inferior and nasal RNFL measurements. This was consistent with a similar study done in India where they also found a negative correlation between age and macular thickness measurements, except for the central foveal thickness. In contrast, in the study done by Grover et al., age had no effect on macular thickness measurements; however, this study was limited by a relatively smaller sample size. ${ }^{15}$ There is conflicting evidence in literature as to whether age can affect RNFL thickness. ${ }^{10}$ Akashi et al. observed that RNFL thickness decreased with age. ${ }^{11}$ This thinning may be explained by the histologic evidence of a decrease in the number of ganglion cells, photoreceptors and retinal pigment epithelial cells that come with age. ${ }^{20}$ Since the abovementioned retinal thickness measurements in our study were less affected by age, they might be better SD-OCT measurement parameters among myopic Filipino patients to monitor for disease progression.

The linear regression models were used to provide reference values for each of the retinal thickness parameters. However, this may be limited by the sample size and a larger population is needed in order to provide a more accurate normative data for Filipino myopic individuals. Future studies with a larger sample size may be done in order to establish an accepted normative range for myopic individuals. Be that as it may, the derived mean values may be a more accurate reference point in the clinical setting rather than the built-in normative values with the OCT machines. This study also reveals the importance of structural peculiarities of the myopic eye and that clinicians should not readily interpret abnormal OCT findings indicated by the machine as outside of the normal limits.

\section{Conclusion}

The central foveal subfield thickness reference values derived from the mean of a normal cohort for each degree of myopia were as follows: low, $264 \pm 24 \mu \mathrm{m}$; moderate, $258 \pm 17 \mu \mathrm{m}$; high, $253 \pm 25 \mu \mathrm{m}$ and pathologic, $218 \pm 48 \mu \mathrm{m}$. For global average RNFL thickness, the following reference values were derived: low, $105.62 \pm 3.89 \mu \mathrm{m}$; moderate, $97.60 \pm 2.45 \mu \mathrm{m}$; high, $85.90 \pm 3.87 \mu \mathrm{m}$ and pathologic, $75.14 \pm 3.89 \mu \mathrm{m}$. Most of the macular thickness and RNFL measurement parameters were affected by the degree of myopia and age, while gender was not a factor. Generally, thickness measurements decreased with increasing level of myopia as well as age. Therefore, it is important to take into account these factors when analyzing retinal thickness measurements among myopic patients. Since the central foveal, temporal parafoveal, nasal perifoveal, inferior RNFL and nasal RNFL thicknesses were less influenced by age, they may be better SD-OCT measurement parameters to monitor for disease among myopic Filipino patients. 
Lastly, using derived mean measurements for each level of myopia and age from a population of the same ethnicity may be a more accurate reference value in the clinical setting than the built-in normative values in OCT machines.

\section{References}

1. Wang Q, Klein BE, Klein R, Moss SE. Refractive status in the Beaver Dam Eye Study. Invest Ophthalmol Vis Sci. 1994;35:4344-4347. https://www.ncbi.nIm.nih.gov/pubmed/8002254.

2. Zhao J, Pan X, Sui R, Munoz SR, Sperduto RD, Ellwein LB. Refractive error study in children: results from Shunyi District, China. Am J Ophthalmol. 2000;129:427-435. https://www.ncbi.nlm.nih.gov/ pubmed/10764849

3. Lin LL, Shih YF, Hsiao CK, Chen CJ, Lee LA, Hung PT. Epidemiologic study of the prevalence and severity of myopia among schoolchildren in Taiwan in 2000. J Formos Med Assoc. 2001;100: 684-691. https://www.ncbi.nlm.nih.gov/pubmed/11760374.

4. Ling SL, Chen AJ, Rajan U, Cheah WM. Myopia in ten year old children-a case control study. Singapore Med J. 1987;28:288-292. https://www.ncbi.nlm.nih.gov/pubmed/3423791.

5. Reyes KB, Uy HS. Refractive errors in Filipino eyes in a single-center study population. Philipp J Ophthalmol. 2010;35:50-55. paojournal.com/vol35no2/toc.php.

6. Kang SH, Hong, SW, Im SK, Lee SH, Ahn MD. Effect of myopia on the thickness of the retinal nerve fiber layer measured by Cirrus HD optical coherence tomography. Invest Ophthalmol Vis Sci. 2010;51:4075-4083. https://www.ncbi.nlm.nih.gov/pubmed/20237247.

7. Foster PJ, Jiang Y. Epidemiology of myopia. Eye (Lond). 2014;28:202-208. https://www.ncbi.nlm. nih.gov/pubmed/24406412.

8. Emerole CG, Nneli RO, Osim EE. Prevalence, distribution and determinants of myopia in Owerri, Nigeria. J Exp Clin Anat. 2013;12: 57-61. http://www.jecajournal.org/article. asp?issn=1596-2393; year=2013; volume=12;issue=2;spage=57;epage=61; aulast=Emerole.

9. Saw SM, Gazzard G, Shi-Yen E, Chua WH. Myopia and associated pathological complications. Ophthal Physio Opt. 2005;25:381-391. https://www.ncbi.nlm.nih.gov/pubmed/16101943.

10. Shoji T, Sato H, Ishida M, Takeuchi M, Chihara E. Assessment of glaucomatous changes in subjects with high myopia using spectral domain optical coherence tomography. Invest Ophthalmol Vis Sci. 2011;52:1098-1102. https://www.ncbi.nlm.nih.gov/pubmed/21051712.

11. Akashi A, Kanamori A, Ueda K, Inoue Y, Yamada Y, Nakamura M. The ability of SD-OCT to differentiate early glaucoma with high myopia from highly myopic controls and non-highly myopic control. Invest Ophthalmol Vis Sci. 2015;56:6573-6580. https://iovs.arvojournals.org/ article.aspx?articleid=2464683.

12. Holden B, Fricke T, Wilson D, et al. Global prevalence of myopia and high myopia and temporal trends from 2000 through 2050. Am J Ophthalmol. 2016;123:1036-1042. https://www.ncbi.nlm. nih.gov/pubmed/26875007.

13. Jonas JB, Xu L. Histological changes of high axial myopia. Eye. 2014;28:113-117. https://www. ncbi.nlm.nih.gov/pmc/articles/PMC3930258.

14. Beresford J, Crewther S, Kiely P, Crewther DP. Comparison of refractive state and circumferential morphology of retina, choroid and sclera in chick models of experimentally induced ametropia. Optom Vis Sci. 2002;78:40-49. https://www.ncbi.nlm.nih.gov/pubmed/11233334.

15. Grover S, Murthy R, Brar V, Chalam KV. Normative data for macular thickness by high-definition spectral-domain optical coherence tomography (Spectralis ${ }^{\circledR}$ ). Am J Ophthalmol. 2009;148:266271. https://www.ncbi.nlm.nih.gov/pubmed/19427616.

16. Kanai K, Abe T, Murayama K, Yoneya S. Retinal thickness and changes with age. Nippon Ganka Gakkai Zasshi. 2002;106:162-165. https://www.ncbi.nlm.nih.gov/pubmed/11925953.

17. Chan A, Duker JS, Ko TH, Fujimoto JG, Schuman JS. Normal macular thickness measurements in healthy eyes using Stratus optical coherence tomography. Arch Ophthalmol. 2006;124:193-198. https://www.ncbi.nlm.nih.gov/pmc/articles/PMC1941772/. 